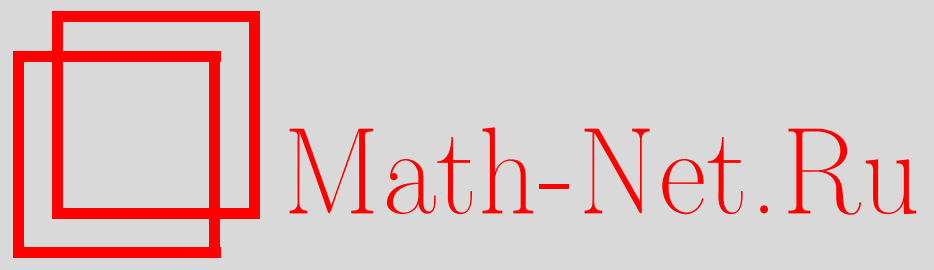

А. А. Боровков, Оценки момента разладки по большим выборкам при неизвестных распределениях, Теория вероятн. и ее примен., 2008, том 53, выпуск $3,437-457$

DOI: https://doi.org/10.4213/tvp2441

Использование Общероссийского математического портала MathNet.Ru подразумевает, что вы прочитали и согласны с пользовательским соглашением

http://www . mathnet.ru/rus/agreement

Параметры загрузки:

IP : 3.85 .5 .30

26 апреля 2023 г., 15:55:42

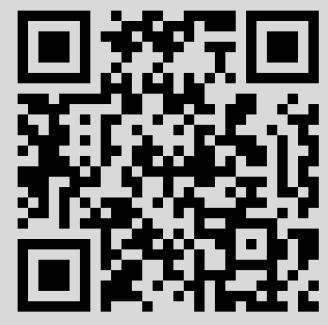




\title{
ДИСКРЕТНОЕ ВРЕМЯ
}

(c) 2008 г.

\author{
БОРОВКОВ А. А.*
}

\section{ОЦЕНКИ МОМЕНТА РАЗЛАДКИ ПО БОЛЬШИМ ВЫБОРКАМ ПРИ НЕИЗВЕСТНЫХ РАСПРЕДЕЛЕНИЯХ ${ }^{1)}$}

\begin{abstract}
Пусть дана выборка $\mathrm{X}=\left(\mathrm{x}_{1}, \mathrm{x}_{2}, \ldots, \mathrm{x}_{n}\right)$, состоящая из $n$ независимых наблюдений в произвольном измеримом пространстве $\mathscr{X}$ и устроенная следующим образом: первые $\theta$ наблюдений имеют распределение $F$, остальные $n-\theta$ наблюдений - распределение $G \neq F$, при этом распределения $F$ и $G$ нам неизвестны, $n$ и $\theta$ велики. В [1] были построены оценки $\theta^{*}$ момента разладки $\theta$ с собственной величиной погрешности (т.е. такие, что $\mathbf{P}_{\theta}\left\{\left|\theta^{*}-\theta\right|>k\right\}$ сходится к нулю с ростом $k$ ) в предположении, что существует известная функция $h$, для которой средние значения $h\left(\mathrm{x}_{j}\right)$ относительно распределений $F$ и $G$ различны. Там же построены последовательные процедуры. В предлагаемой работе получены аналогичные результаты при ослаблении названного выше предположения или даже при его отсутствии. Один вариант ослабления состоит в предположении, что существуют известные функции $h_{1}, h_{2}, \ldots, h_{l}$ на $\mathscr{X}$ такие, что хотя бы для одной из них средние значения $h_{j}\left(\mathrm{x}_{i}\right)$ относительно распределений $F$ и $G$ различны. Другой вариант ослабления не предполагает существования известных нам функций $h_{j}$, но допускает возможность оценивать неизвестные распределения $F$ и $G$ по начальной и конечной частям выборки X. Рассмотрены также последовательные процедуры.
\end{abstract}

Ключевые слова и фразы: задача о разладке при неизвестных распределениях, момент разладки, последовательное оценивание.

\section{1. Оценки момента разладки по всей выборке}

1.1. Введение. Пусть дана выборка

$$
\mathrm{X}=\left(\mathrm{x}_{1}, \mathrm{x}_{2}, \ldots, \mathrm{x}_{n}\right)
$$

* Институт математики им. С. Л. Соболева СО РАН, просп. Академика Коптюга, 4, 630090 Новосибирск, Россия; Новосибирский государственный университет, ул. Пирогова, 2, 630090 Новосибирск, Россия; e-mail: borovkov@math.nsc.ru

1) Работа выполнена при поддержке РФФИ (проект №05-01-00810) и программы «Ведушие научные школы» (проект НШ-8980-2006.1). 
состоящая из $n$ независимых наблюдений в произвольном измеримом пространстве $\mathscr{X}$. Относительно элементов выборки Х мы предполагаем, что первые $\theta$ наблюдений имеют распределение $F$, остальные $n-\theta$ наблюдений - распределение $G \neq F$, при этом распределения $F$ и $G$ нам не известны, а значения $\theta, n-\theta$ неограниченно растут при $n \rightarrow \infty$. Задаче об оценке $\theta^{*}$ момента разладки $\theta$ по всей выборке $\mathrm{X}$ при неизвестных распределениях $F$ и $G$ посвящено значительное количество работ (см., например, [2]-[9]). Краткий обзор результатов из [2]-[9] содержится в [1]. В [8], как и в [1], содержатся также результаты, относящиеся к последовательным процедурам. Более подробные комментарии по поводу результатов работы [8] содержатся в пп. 1.4, 2.4 .

Условие $F \neq G$ в [1] трактуется как существование известной нам функции $h$ такой, что $\mathbf{E} h\left(\mathrm{x}^{(F)}\right) \neq \mathbf{E} h\left(\mathrm{x}^{(G)}\right)$ (здесь и далее $\mathbf{x}^{(H)}$ обозначает случайную величину с распределением $H(H$ принимает значения $F$ или $G)$ ). При выполнении этого условия в [1] построены оценки $\theta^{*}$ и получены неравенства для $\mathbf{P}_{\theta}\left\{\left|\theta^{*}-\theta\right|>k\right\}$, правые части которых найдены в явном виде (они зависят, естественно, от свойств распределений $F$ и $G$ ).

Цель настоящей работы - получить аналогичные результаты при ослаблении названного выше условия о сушествовании известной функции $h$ или даже при полном отказе от него.

Ослабление этого условия имеет следующий вид.

$\left[\mathbf{h}_{\mathbf{1}}, \ldots, \mathbf{h}_{\mathbf{l}}\right]$. Существуют известние нам $l$ измеримьх функиий $h_{1}, \ldots, h_{l}$, для которых соотношение $F \neq G$ влечет за собой выполнение по крайней мере одного из неравенств

$$
\mathbf{E} h_{j}\left(\mathrm{x}^{(F)}\right) \neq \mathbf{E} h_{j}\left(\mathrm{x}^{(G)}\right), \quad j=1, \ldots, l .
$$

Например, в случае нормальных (или близких к ним) распределений $F$ и $G$, когда известно лишь, что $F \neq G$, невозможно указать одну функцию $h$ такую, что $F \neq G$ влечет $\mathbf{E} h\left(\mathrm{x}^{(F)}\right) \neq \mathbf{E} h\left(\mathrm{x}^{(G)}\right)$. Но если взять две функции $h_{1}(x)=x, h_{2}(x)=x^{2}$, то с необходимостью соотношение $F \neq G$ влечет за собой выполнение $\left[\mathbf{h}_{\mathbf{1}}, \mathbf{h}_{\mathbf{2}}\right]$ и это условие перестает быть ограничением. Аналогичная ситуация может иметь место и для других параметрических семейств, например, для семейств равномерных распределений, гамма-распределений и др. Если мы имеем дело с $k$-мерными нормальными распределениями, $k>1$, то для того, чтобы «различать» $F$ и $G$, надо взять $l=k+k(k+1) / 2$ функций $(k$ линейных и $k(k+1) / 2$ квадратичных, соответствующих первым и вторым моментам).

Задачу можно поставить и несколько иначе. При наблюдении за выборкой X нас может интересовать не возможность изменения распределения, а нечто меньшее - возможность изменения лишь некоторого 
количества характеристик вида $\mathrm{E} h_{j}\left(\mathrm{x}_{i}\right)$, где $h_{j}(\cdot)$ - заданные функции. Мы приходим, таким образом, к следующей постановке задачи: даны известные измеримые функции $h_{j}: \mathscr{X} \rightarrow \mathbf{R}, j=1, \ldots, l$, такие, что в момент $\theta$ меняется хотя бы одно из значений $\mathbf{E} h_{j}\left(\mathrm{x}_{i}\right)$. Задача, по-прежнему, состоит в оценивании параметра $\theta$. Очевидно, что в момент времени $\theta$ с необходимостью будет меняться и распределение (но не наоборот). Таким образом, нас интересуют такие изменения распределения, при которых найдется хотя бы одно $j \in\{1, \ldots, l\}$, для которого

$$
\mathbf{E} h_{j}\left(\mathrm{x}^{(F)}\right) \neq \mathbf{E} h_{j}\left(\mathrm{x}^{(G)}\right) \text {. }
$$

Отметим, что значение $j$ из (1) (возможно, не единственное) нам не известно.

Можно вообще отказаться от условий вида $\left[\mathbf{h}_{\mathbf{1}}, \ldots, \mathbf{h}_{\mathbf{l}}\right]$. В качестве альтернативного условия можно предполагать возможность оценивать неизвестные распределения $F$ и $G$ (или функционалы от них) по начальной и конечной частям выборки Х. Именно, мы можем предполагать, что существует известная нам последовательность

$$
M=M(n)=o(n), \quad M \rightarrow \infty \quad \text { при } \quad n \rightarrow \infty,
$$

такая, что

$$
M<\theta<n-M
$$

при всех достаточно больших $n$. Это условие, очевидно, всегда выполняется, если

$$
\frac{\theta}{n} \rightarrow \rho \in(0,1) \quad \text { при } n \rightarrow \infty .
$$

Это последнее условие предполагается выполненным во многих работах (cp. с [2]-[9]).

Несколько более общее условие состоит в предположении, что $\theta-$ случайная величина (байесовский подход) такая, что для распределения $P$ этой величины выполняется условие

$$
[\mathbf{P}]: \quad P_{(M)}:=P[1, M] \rightarrow 0, \quad P^{(M)}:=P[n-M, n] \rightarrow 0
$$

при $n \rightarrow \infty$, где $M=o(n), M \rightarrow \infty$.

Отметим, что условия $\theta \rightarrow \infty, n-\theta \rightarrow \infty$ присутствуют во всех утверждениях и в известном смысле являются необходимыми и, стало быть, не ограничивают общности. Отказ от этих условий делает задачу о моменте разладки при неограниченно растущих $n$ малосодержательной. Условия (2), [P] суть лишь некоторая спецификация условий $\theta \rightarrow \infty$, $n-\theta \rightarrow \infty$, предполагающая знание нужной нам последовательности $M$. 
1.2. Основные результаты об оценивании $\theta$ по всей выборке $\mathrm{X}$ при выполнении условия $\left[\mathbf{h}_{1}, \ldots, \mathbf{h}_{1}\right]$. Пусть выполнено условие $\left[\mathbf{h}_{\mathbf{1}}, \ldots, \mathbf{h}_{\mathbf{l}}\right]$. Положим

$$
\begin{gathered}
\mathrm{y}_{i}^{(j)}=h_{j}\left(\mathrm{x}_{i}\right), \quad S_{l}^{(j)}=\sum_{i=1}^{l} \mathrm{y}_{i}^{(j)}, \\
\mathrm{y}^{(H, j)}=h_{j}\left(\mathrm{x}^{(H)}\right), \quad a^{(H, j)}=\mathbf{E} h_{j}\left(\mathrm{x}^{(H)}\right), \quad \mathrm{y}^{(H, j, 0)}=\mathrm{y}^{(H, j)}-a^{(H, j)}, \\
Q_{j}(t)=\sum_{i=1}^{t}\left(\mathrm{y}_{i}^{(j)}-\frac{S_{t}^{(j)}}{t}\right)^{2}+\sum_{i=t+1}^{n}\left(\mathrm{y}_{i}^{(j)}-\frac{S_{n}^{(j)}-S_{t}^{(j)}}{n-t}\right)^{2} .
\end{gathered}
$$

В [1] в качестве одного из подходов к оцениванию $\theta$ в случае $l=1$ был рассмотрен прием, состоящий в наблюдении за траекторией $Q_{1}(t)$ и выборе в качестве $\theta$ значения $t$, при котором достигается наименьшее значение $Q_{1}(t)$. При $l>1$ естественно рассматривать аналог этого подхода, когда наблюдается $l$ траекторий $Q_{1}(t), \ldots, Q_{l}(t)$ и в качестве оценки параметра $\theta$ выбирается значение

$$
\theta^{*}=\underset{t}{\arg \min } Q(t), \quad Q(t):=\sum_{k=1}^{l} Q_{k}(t),
$$

где достигается «усредненный» минимум.

Ниже мы найдем оценки вероятностей отклонений $\theta^{*}$ от $\theta$ при некоторых дополнительных предположениях относительно распределений $F$ и $G$, касающихся поведения «хвостов» этих распределений.

1.2.1. Сначала рассмотрим случай, когда распределения велииин $\mathrm{y}^{(H, j)}$ удовлетворяют условию Крамера.

Говорят, что случайная величина $\xi$ удовлетворяет двустороннему условию Крамера, если в окрестности точки $\lambda=0$ выполняется

$$
\varphi(\lambda)=\mathbf{E} e^{\lambda \xi}<\infty
$$

Через $\mathbf{P}_{\theta}$ обозначим распределение в пространстве последовательностей $\mathrm{X}$ при фиксированном значении $\theta$ и положим

$$
\mathrm{y}^{(H)}=\sum_{j=1}^{l} \mathrm{y}^{(H, j)} .
$$

Теорема 1. Пусть выполнено условие (1) и $\delta(t)-$ любая фиксированная функиия такая, что $\delta(t) \rightarrow 0$ nри $t \rightarrow \infty$. Тогда:

1) если случайная величина $\mathrm{y}^{(G)}$ удовлетворяет двустороннему условию Крамера, то существует число $\varphi_{1}<1$ (оно определено в доказательстве), при котором справедливо неравенство

$$
\mathbf{P}_{\theta}\left\{\theta^{*}-\theta>k\right\} \leqslant 2 \varphi_{1}^{k}[1+o(1)] \quad \text { npu } \quad \theta \rightarrow \infty, \quad n-\theta \rightarrow \infty
$$


2) если случайная величина $\mathrm{y}^{(F)}$ удовлетворяет двустороннему условию Крамера, то существует число $\varphi_{2}<1$, при котором

$$
\mathbf{P}_{\theta}\left\{\theta^{*}-\theta<-k\right\} \leqslant 2 \varphi_{2}^{k}[1+o(1)] \quad \text { npu } \quad \theta \rightarrow \infty, \quad n-\theta \rightarrow \infty .
$$

Оченки о(1) в приведенных неравенствах являются равномерными по всем $k>0, k \leqslant \theta \delta(\theta), k \leqslant(n-\theta) \delta(n-\theta)$.

Док аз а т ль с т в П. Положим

$$
\begin{aligned}
& \mathrm{z}^{(G, j)}=2 \mathrm{y}^{(G, j, 0)}\left(a^{(F, j)}-a^{(G, j)}\right)-\left(a^{(F, j)}-a^{(G, j)}\right)^{2}, \\
& \mathrm{z}^{(F, j)}=2 \mathrm{y}^{(F, j, 0)}\left(a^{(F, j)}-a^{(G, j)}\right)+\left(a^{(F, j)}-a^{(G, j)}\right)^{2}, \\
& \mathrm{z}^{(G)}=\sum_{j=1}^{l} \mathrm{z}^{(G, j)}, \quad \mathrm{z}^{(F)}=\sum_{j=1}^{l} \mathrm{z}^{(F, j)},
\end{aligned}
$$

где ввиду условия (1) выполнены неравенства $\mathrm{Ez}^{(F)}>0$ и $\mathbf{E z}^{(G)}<0$.

Отметим, что если $\mathrm{y}^{(H)}$ удовлетворяет условию Крамера (6), то этому же условию удовлетворяет и ${ }^{(H)}$. Отметим также, что если для случайной величины $\xi$ выполнено условие Крамера (6) и $a=\mathbf{E} \xi<0$, то $\varphi^{\prime}(0)=a<0, \varphi:=\min _{\lambda \geqslant 0} \varphi(\lambda)<1$ и этот минимум всегда достигается.

Для величины $Q(t)$, определенной в $(5)$, имеем

$$
Q(t)=\sum_{j=1}^{l} \sum_{i=1}^{n}\left(\mathrm{y}_{i}^{(j)}\right)^{2}-\widetilde{Q}(t)
$$

где

$$
\widetilde{Q}(t)=\sum_{j=1}^{l} \widetilde{Q}^{(j)}(t), \quad \widetilde{Q}^{(j)}(t)=\frac{\left(S_{t}^{(j)}\right)^{2}}{t}+\frac{\left(S_{n}^{(j)}-S_{t}^{(j)}\right)^{2}}{n-t} .
$$

Таким образом, $\theta^{*}=\arg \max _{t} \widetilde{Q}(t)$. Для $k>0$ и $\mathrm{y}_{i}^{(j, 0)}=\mathrm{y}_{i}^{(j)}-\mathbf{E y}_{i}^{(j)}$ имеем

$$
\begin{gathered}
S_{\theta+k}^{(j)}=\sum_{i=1}^{\theta} \mathrm{y}_{i}^{(j, 0)}+\theta a^{(F, j)}+\sum_{i=\theta+1}^{\theta+k} \mathrm{y}_{i}^{(j, 0)}+k a^{(G, j)} \\
S_{n}^{(j)}-S_{\theta+k}^{(j)}=\sum_{i=\theta+1}^{n} \mathrm{y}_{i}^{(j, 0)}+(n-\theta) a^{(G, j)}-\sum_{i=\theta+1}^{\theta+k} \mathrm{y}_{i}^{(j, 0)}-k a^{(G, j)} .
\end{gathered}
$$

Кроме того, справедливы разложения:

$$
\frac{1}{\theta+k}=\frac{1}{\theta}\left(1+\frac{k}{\theta}\right)^{-1}=\frac{1}{\theta}-\frac{k}{\theta^{2}}+o\left(\frac{k}{\theta^{2}}\right)
$$

при $k \leqslant \theta \delta(\theta), \theta \rightarrow \infty$ и и

$$
\frac{1}{n-\theta-k}=\frac{1}{n-\theta}\left(1-\frac{k}{n-\theta}\right)^{-1}=\frac{1}{n-\theta}+\frac{k}{(n-\theta)^{2}}+o\left(\frac{k}{(n-\theta)^{2}}\right)
$$


при $k \leqslant(n-\theta) \delta(n-\theta), n-\theta \rightarrow \infty$. Используя представления $(7),(8)$ и $(9)$, получаем

$$
\widetilde{Q}(\theta+k)=Q_{0}+Z_{k}+\varepsilon
$$

где

$$
Z_{k}=2 \sum_{i=1}^{k} \sum_{j=1}^{l}\left(a^{(F, j)}-a^{(G, j)}\right) \mathrm{y}_{\theta+i}^{(j, 0)}-k \sum_{j=1}^{l}\left(a^{(F, j)}-a^{(G, j)}\right)^{2}
$$

а значение

$$
Q_{0}=\sum_{j=1}^{l}\left(\sum_{i=1}^{\theta} \mathrm{y}_{i}^{(j, 0)}+\theta a^{(F, j)}\right)^{2} \frac{1}{\theta}+\sum_{j=1}^{l}\left(\sum_{i=\theta+1}^{n} \mathrm{y}^{(j, 0)}+(n-\theta) a^{(G, j)}\right)^{2} \frac{1}{n-\theta}
$$

не зависит от $k, \varepsilon=\varepsilon(k, \theta, n) \stackrel{p}{\rightarrow} 0$ при $\theta \rightarrow \infty$ и $n-\theta \rightarrow \infty$. Следовательно, согласно (5), (10), (11), $\theta^{*}-\theta$ есть значение $k$, которое максимизирует $Z_{k}+\varepsilon$ :

$$
\theta^{*}-\theta=\underset{k}{\arg \max }\left(Z_{k}+\varepsilon\right)
$$

при этом суммы $Z_{k}$ равны суммам независимых одинаково распределенных случайных величин, совпадающих по распределению со случайной величиной $z^{(G)}, \mathbf{E z}_{z}^{(G)}<0$. Ввиду представления (12), утверждение п. 1) теоремы 1 следует из леммы 1 в [1] при $\varphi_{1}=\min _{\lambda} \varphi^{(G)}(\lambda)<1$, $\varphi^{(G)}(\lambda)=\mathbf{E} e^{\lambda z^{(G)}}$.

Второе утверждение теоремы доказывается аналогично при $\varphi_{2}=$ $\min _{\lambda} \varphi^{(F)}(\lambda)<1$.

1.2.2. Рассмотрим теперь случай, когда условие Kрамера $\partial л я \mathrm{y}^{(H)}$ не выполнено.

Функция $V(t)$ называется правильно меняющейся при $t \rightarrow \infty$, если она представима в виде $V(t)=t^{-\alpha} L_{V}(t)$, где $L_{V}(t)$ - медленно меняющаяся при $t \rightarrow \infty$ функция. В дальнейшем, не оговаривая это особо, мы будем считать, что $\alpha>1$.

Теорема 2. Пусть выполнено условие (1), оченка $\theta^{*}$ определена в (5). Тогда:

1) если $\mathbf{P}\left\{\left|\mathrm{y}^{(G)}\right|>t\right\} \leqslant V(t)$ для некоторой правильно меняющейся мажоранты $V(t) \equiv t^{-\alpha} L_{V}(t)$, то существует постоянная $c_{1}<\infty$ такая, что справедливо неравенство

$$
\mathbf{P}_{\theta}\left\{\theta^{*}-\theta \geqslant k\right\} \leqslant k c_{1} V(k)
$$

nрu $\theta \rightarrow \infty u n-\theta \rightarrow \infty$;

2) если $\mathbf{P}\left\{\left|\mathrm{y}^{(F)}\right|>t\right\} \leqslant W(t)$ для некоторой правильно меняюшейся мажоранты $W(t) \equiv t^{-\beta} L_{W}(t)$, то существует постоянная $c_{2}<\infty$ такая, что при $\theta \rightarrow \infty$ и $n-\theta \rightarrow \infty$ выполняется неравенство

$$
\mathbf{P}_{\theta}\left\{\theta^{*}-\theta \leqslant-k\right\} \leqslant k c_{2} W(k) .
$$


Д о к а за тель с т в о т е о р е м 2 совершенно аналогично доказательству теоремы 1 и теоремы 8 в [1] с использованием леммы 2 в [1]. Это доказательство приводит к неравенству (13), в котором вместо $c_{1}$ стоит множитель

$$
\left(1+\frac{1}{2(\alpha+1)}\right)\left(\frac{A}{2}\right)^{-\alpha}(1+\varepsilon(k))(1+o(1)),
$$

где $A=\sum_{j=1}^{l}\left(a^{(F, j)}-a^{(G, j)}\right)^{2}, \varepsilon(k) \rightarrow 0$ при $k \rightarrow \infty$, остаточный член $o(1)$ равномерен по всем $k>0, k \leqslant \delta(\theta) \theta, k \leqslant(n-\theta) \delta(n-\theta) ; \delta(t)$ - любая фиксированная функция такая, что $\delta(t) \rightarrow 0$ при $t \rightarrow \infty$. Ясно, что полученное утверждение влечет за собой (13). Неравенство (14) получается аналогично.

1.3. Оценки параметра $\theta$ по всей выборке $\mathrm{X}$ по сгруппированным данным. В условии $\left[\mathbf{h}_{1}, \ldots, \mathbf{h}_{\mathbf{l}}\right]$, использованном в предыдущем пункте, в качестве функций $h_{j}$ могут быть взяты моментные функции, гармоники и другие функции, способные при достаточном их количестве «различать» распределения $F$ и $G$. В этом пункте мы остановимся на индикаторных функциях $h_{j}$, что соответствует группировке наблюдений. Мы будем предполагать, что выполнено следующее условие.

$\left[\mathscr{X}_{1}, \ldots, \mathscr{X}_{l}\right]$. Cуществует известное нам разбиение пространства $\mathscr{X}$ на непересекаюшиеся подмножества $\mathscr{X}_{1}, \ldots, \mathscr{X}_{l}, \bigcup_{j=1}^{l} \mathscr{X}_{j}=\mathscr{X}$, makoe, чmo

$$
\left\{p_{j}^{(F)}\right\} \neq\left\{p_{j}^{(G)}\right\}, \quad \text { 2дe } \quad p_{j}^{(H)}=H\left(\mathscr{X}_{j}\right)>0
$$

H принимает значения $F$ и $G$.

Условие $\left[\mathscr{X}_{1}, \ldots, \mathscr{X}_{l}\right]$ является частным случаем условий $\left[\mathbf{h}_{\mathbf{1}}, \ldots, \mathbf{h}_{\mathbf{l}}\right]$ и соответствует индикаторным функциям $h_{j}(\mathrm{x})=I_{\mathscr{X}_{j}}(\mathrm{x})$. Поэтому для отыскания оценок $\theta^{*}$ мы можем воспользоваться теоремой 1 (условие Крамера для ${ }^{(H)}$ в этом случае всегда выполнено). Однако если выполнено условие (2), то мы можем воспользоваться несколько иным подходом.

\section{4. Оценки параметра $\theta$ по сгруппированным данным при} выполнении условия (2). Если выполнено условие (2), то мы можем использовать первые и последние $M$ элементов выборки для оценки неизвестных распределений $F$ и $G$ с тем, чтобы получить подходящую «эмпирическую» функцию $h_{1}^{*}(\mathrm{x})$, зная которую мы могли бы поступать так же, как в случае выполнения условия $\left[\mathbf{h}_{\mathbf{1}}\right]$ (см. [1]).

Итак, пусть выполнены условия $\left[\mathscr{X}_{1}, \ldots, \mathscr{X}_{l}\right]$ и $(2)$. Пусть $\nu_{j}^{(F)}$, $\nu_{j}^{(G)}$ - частоты попадания наблюдений в $\mathscr{X}_{j}$ соответственно в первых и последних $M$ элементах выборки,

$$
p_{j}^{*(H)}=\frac{\nu_{j}^{(H)}}{M} .
$$


Заметим, далее, что в силу (15)

$$
s_{1}:=\sum_{j=1}^{l} p_{j}^{(F)} \ln \frac{p_{j}^{(F)}}{p_{j}^{(G)}}>0, \quad s_{2}:=\sum_{j=1}^{l} p_{j}^{(G)} \ln \frac{p_{j}^{(F)}}{p_{j}^{(G)}}<0 .
$$

Поэтому при любых $\varepsilon_{j}^{(H)}$ таких, что $\left|\varepsilon_{j}^{(H)}\right| \leqslant \varepsilon$, и достаточно малых $\varepsilon$ будут также выполнены неравенства

$$
\begin{aligned}
& \sum_{j=1}^{l} p_{j}^{(F)} \ln \frac{p_{j}^{(F)}\left(1+\varepsilon_{j}^{(F)}\right)}{p_{j}^{(G)}\left(1+\varepsilon_{j}^{(G)}\right)}=s_{1}+O(\varepsilon)>0 \\
& \sum_{j=1}^{l} p_{j}^{(G)} \ln \frac{p_{j}^{(F)}\left(1+\varepsilon_{j}^{(F)}\right)}{p_{j}^{(G)}\left(1+\varepsilon_{j}^{(G)}\right)}=s_{2}+O(\varepsilon)<0 .
\end{aligned}
$$

В связи с этим естественно ожидать, что для функции

$$
h^{*}(\mathrm{x})=\ln \frac{p_{j}^{*(F)}}{p_{j}^{*(G)}} \quad \text { при } \quad \mathrm{x} \in \mathscr{X}_{j}
$$

с высокой вероятностью будут выполняться неравенства

$$
\mathrm{E}\left(h^{*}\left(\mathrm{x}^{(F)}\right) \mid \mathscr{F}\right)>\frac{s_{1}}{2}>0, \quad \mathbf{E}\left(h^{*}\left(\mathrm{x}^{(G)}\right) \mid \mathscr{F}\right)<-\frac{s_{2}}{2}<0
$$

где $\mathscr{F}-\sigma$-алгебра, порожденная подвыборками $\mathrm{X}_{(M)}:=\left(\mathrm{x}_{1}, \ldots, \mathrm{x}_{M}\right)$ и $\mathrm{X}^{(M)}:=\left(\mathrm{x}_{n-M+1}, \ldots, \mathrm{x}_{n}\right), \mathrm{x}^{(F)}$ и $\mathrm{x}^{(G)}$ не зависят от $\mathscr{F}$.

Мы можем перейти теперь к описанию процедуры построения оценки. Пусть выполнено условие (2). Рассмотрим новую выборку $\mathrm{X}_{(M)}^{(M)}:=\left(\mathrm{x}_{M+1}, \ldots, \mathrm{x}_{n-M}\right)$ и суммы $S_{k}=\sum_{i=1}^{k} h^{*}\left(\mathrm{x}_{M+i}\right)$. В качестве оценки $\theta^{*}$ возьмем значение

$$
\theta^{*}=M+\min \left\{k \leqslant n-2 M: S_{k}=\bar{S}_{n-2 M}\right\}
$$

где $\bar{S}_{r}=\max _{k \leqslant r} S_{k}$. Такого рода оценки (при неслучайной функции $h^{*}$, удовлетворяющей (16) (или условию $\left(D_{0}\right)$ в [1]) были изучены в теореме $1[1]$.

Теорема 3. Пусть вынолнены условия $\left[\mathscr{X}_{1}, \ldots, \mathscr{X}_{l}\right]$ u (2). Тогда для всех $k, 0 \leqslant k<n-2 M-\theta$, справедливо неравенство

$$
\mathbf{P}\left\{\theta^{*}-\theta>k\right\} \leqslant q_{M}+2\left[\varphi_{(G)}+O(\varepsilon)\right]^{k}
$$

и для всех $k, 0 \leqslant k<\theta$, справедливо неравенство

$$
\mathbf{P}\left\{\theta^{*}-\theta<-k\right\} \leqslant q_{M}+2\left[\varphi_{(F)}+O(\varepsilon)\right]^{k},
$$


где $\varepsilon=M^{-1 / 4} \rightarrow 0$,

$$
\begin{gathered}
\varphi_{(H)}=\min _{\lambda} \sum_{j=1}^{l} p_{j}^{(H)}\left(\frac{p_{j}^{(F)}}{p_{j}^{(G)}}\right)^{\lambda}<1, \\
q_{M} \leqslant 4 l e^{-b \sqrt{M}(1+o(1))}, \quad b=\min _{j, H} \frac{p_{j}^{(H)}}{2\left(1-p_{j}^{(H)}\right)} .
\end{gathered}
$$

Д о к а з а т е л ь с т в о. Обозначим через $\omega$ элементарное событие из $\mathscr{F}$ и положим при $\varepsilon=M^{-1 / 4}$

$$
\Omega_{M, H}=\left\{\omega: \max _{j \leqslant l}\left|p_{j}^{*(H)}-p_{j}^{(H)}\right|>\varepsilon p_{j}^{(H)}\right\}, \quad \Omega_{M}=\Omega_{M, F} \cup \Omega_{M, G} .
$$

Тогда

$$
\mathbf{P}\left\{\theta^{*}-\theta>k\right\} \leqslant q_{M}+\mathbf{E}\left(\mathbf{P}\left\{\theta^{*}-\theta>k \mid \mathscr{F}\right\} ; \bar{\Omega}_{M}\right),
$$

где $q_{m}=\mathbf{P}\left(\Omega_{M}\right)$. Рассмотрим сначала второе слагаемое в правой части (20). На множестве $\bar{\Omega}_{M}$, дополнительном к $\Omega_{M}$, выполнено (16). Кроме того, $\theta^{*}$ и $\mathscr{F}$ независимы. Поэтому мы можем воспользоваться теоремой 1 из [1], в силу которой при $\omega \in \bar{\Omega}_{M}, 0 \leqslant k<n-2 M-\theta$ выполнено неравенство

$$
\mathbf{P}\left\{\theta^{*}-\theta>k \mid \mathscr{F}\right\} \leqslant 2 \varphi_{1}^{k},
$$

где

$$
\begin{aligned}
\varphi_{1} & =\min _{\lambda} \mathbf{E}\left(e^{\lambda h^{*}\left(\mathrm{x}^{(G)}\right)} \mid \mathscr{F}\right)=\min _{\lambda} \mathbf{E}\left(\sum_{j=1}^{l} p_{j}^{(G)}\left(\frac{p_{j}^{*(F)}}{p_{j}^{*(G)}}\right)^{\lambda} \mid \mathscr{F}\right) \\
& =\min _{\lambda} \mathbf{E}\left[\left(\sum_{j=1}^{l} p_{j}^{(G)}\left(\frac{p_{j}^{(F)}}{p_{j}^{(G)}}\right)^{\lambda}+O(\varepsilon)\right) \mid \mathscr{F}\right]=\varphi_{(G)}+O(\varepsilon),
\end{aligned}
$$

$O(\varepsilon)$ в правой части равномерно по $\omega \in \bar{\Omega}_{M}, O(\varepsilon)$ в промежуточном равенстве равномерно и по $\lambda$.

Для оценки слагаемого $q_{M}$ в $(20)$ нам понадобится следуюшая лемма.

Лемма 1. При $\varepsilon=M^{-1 / 4}, M \rightarrow \infty$ выполняется неравенство

$$
\mathbf{P}\left\{\max _{j \leqslant l}\left|p_{j}^{*(H)}-p_{j}^{(H)}\right|>\varepsilon p_{j}^{(H)}\right\} \leqslant 2 l e^{-b \sqrt{M}(1+o(1))},
$$

¿de

$$
b=\min _{j, H} \frac{p_{j}^{(H)}}{2\left(1-p_{j}^{(H)}\right)} .
$$


Д о к а з а т е л с с в о. В силу экспоненциального неравенства Чебышёва

$$
\mathbf{P}\left\{\max _{j \leqslant l}\left(p_{j}^{*(H)}-p_{j}^{(H)}\right)>\varepsilon p_{j}^{(H)}\right\} \leqslant \sum_{j=1}^{l} e^{-M \Lambda_{j}^{(H)}\left(p_{j}^{(H)}(1+\varepsilon)\right)}
$$

где

$$
\Lambda_{j}^{(H)}(v)=v \ln \frac{v}{p_{j}^{(H)}}+(1-v) \ln \frac{1-v}{1-p_{j}^{(H)}}
$$

есть функция уклонений случайной величины в схеме Бернулли с вероятностью значения 1 , равной $p_{j}^{(H)}$. Аналогично,

$$
\mathbf{P}\left\{\min _{j}\left(p_{j}^{*(H)}-p_{j}^{(H)}\right)<-\varepsilon p_{j}^{(H)}\right\} \leqslant \sum_{j=1}^{l} e^{-M \Lambda_{j}^{(H)}\left(p_{j}^{(H)}(1-\varepsilon)\right)} .
$$

Функции $\Lambda_{j}^{(H)}$ и их первые производные равны 0 в точке $p_{j}^{(H)}$, а вторые производные положительны, так что при $\varepsilon \rightarrow 0$

$$
\Lambda_{j}^{(H)}\left(p_{j}^{(H)} \pm \varepsilon p_{j}^{(H)}\right)=\frac{\varepsilon^{2} p_{j}^{(H)}}{2\left(1-p_{j}^{(H)}\right)}+O\left(\varepsilon^{3}\right)
$$

Поэтому при $\varepsilon=M^{-1 / 4}, M \rightarrow \infty$

$$
\mathbf{P}\left\{\max _{j \leqslant l}\left|p_{j}^{*(H)}-p_{j}^{(H)}\right|>\varepsilon p_{j}^{(H)}\right\} \leqslant 2 l e^{-M b \varepsilon^{2}(1+O(\varepsilon))}=2 l e^{-b \sqrt{M}(1+o(1))}
$$

где $b$ определено в (23). Лемма доказана.

Сопоставляя (20), (21) и утверждение леммы, мы получим (18). Неравенство (19) доказывается точно так же с помощью теоремы 1 в [1]. Теорема доказана.

3 а м е ч а н и е 1. Если вместо (2) выполнено более широкое условие $[\mathbf{P}]$, то утверждение теоремы 3 , очевидно, сохранится, если в правые части неравенств $(18),(19)$ добавить слагаемые $P_{(M)}+P^{(M)}$.

Несколько иной подход к оцениванию $\theta$ по сгруппированным данным предложен в $[10]$ в предположении, что выполнены условия $\left[\mathscr{X}_{1}, \ldots, \mathscr{X}_{l}\right]$ и

$$
\liminf _{n \rightarrow \infty} \frac{1}{n}(\theta, n-\theta)>0
$$

Строятся отношения

$$
p_{j, k}=\frac{\nu_{j, k}}{k} \quad \text { и } \quad q_{j}^{(n-k)}=\frac{\nu_{j}^{(n-k)}}{n-k}
$$


где $\nu_{j, k}$ и $\nu_{j}^{(n-k)}$ - числа элементов выборок $\left(\mathrm{x}_{1}, \ldots, \mathrm{x}_{k}\right)$ и $\left(\mathrm{x}_{k+1}, \ldots, \mathrm{x}_{n}\right)$ соответственно, попавших в множество $\mathscr{X}_{j}$. Затем рассматриваются суммы $S_{k}=\sum_{i=1}^{k} h_{k}\left(\mathrm{x}_{i}\right)$, где

$$
h_{k}(\mathrm{x})=I_{\mathscr{X}_{j}}(\mathrm{x}) \ln \frac{p_{j, k}}{q_{j}^{(n-k)}},
$$

и в качестве $\theta^{*}$ выбирается значение

$$
\theta^{*}=\min \left\{k \leqslant n: S_{k}=\bar{S}_{n}\right\}, \quad \bar{S}_{n}=\max _{k \leqslant n} S_{k}
$$

Приведена схема доказательства того, что $\left|\theta^{*}-\theta\right|=O_{p}(1)$.

1.5. О построении оценок $\theta$ без предположения $\left[\mathscr{X}_{1}, \ldots, \mathscr{X}_{l}\right]$. Если случайные величины $\mathrm{x}_{i}$ целочисленны и ограничены, скажем, $\mathscr{X}=\{1, \ldots, l\}$, то условие $\left[\mathscr{X}_{1}, \ldots, \mathscr{X}_{l}\right]$, фигурирующее в теореме 3 , выполнено очевидным образом и, стало быть, перестает быть ограничением. Если $\mathscr{X}_{i}$ ограничены, $\mathscr{X}=[-N, N]$, а распределения $F$ и $G$ имеют на $[-N, N]$ положительные плотности, то от условия $\left[\mathscr{X}_{1}, \ldots, \mathscr{X}_{l}\right]$ (или от необходимости строить разбиение $\left.\left\{\mathscr{X}_{1}, \ldots, \mathscr{X}_{l}\right\}\right)$ можно избавиться следующим образом. В качестве функции $h^{*}(\mathrm{x})$, фигурирующей в предыдущем пункте, следует взять $\ln \left(f^{*}(\mathrm{x}) / g^{*}(\mathrm{x})\right)$, где $f^{*}, g^{*}$ - ядерные оценки плотностей $f$ и $g$ для $F$ и $G$ соответственно, построенные с помощью прямоугольных ядер:

$f^{*}(x)=\Delta^{-1}\left(F_{M}(x+\Delta)-F_{M}(x)\right), \quad g^{*}(x)=\Delta^{-1}\left(G^{(M)}(x+\Delta)-G^{(M)}(x)\right)$,

$-N \leqslant x \leqslant N-\Delta$, где $\Delta=\Delta(M) \rightarrow 0, \Delta M \rightarrow \infty$ при $M \rightarrow \infty$ (например, $\left.\Delta=M^{-1 / 2}\right), F_{M}, G^{(M)}$ - эмпирические функции распределения, построенные соответственно по первым и последним $M$ наблюдениям. Аналогично предыдущему можно построить множества

$$
\begin{aligned}
& \Omega_{M, F}=\left\{\max _{-N \leqslant x \leqslant N-\Delta}\left|f^{*}(x)-f(x)\right|>\varepsilon f(x)\right\}, \\
& \Omega_{M, G}=\left\{\max _{-N \leqslant x \leqslant N-\Delta}\left|g^{*}(x)-g(x)\right|>\varepsilon g(x)\right\}
\end{aligned}
$$

так, что $q_{M}=\mathbf{P}\left\{\Omega_{M, F} \cup \Omega_{M, G}\right\} \rightarrow 0$ при $\varepsilon=M^{-1 / 8}, M \rightarrow \infty$. B этом случае утверждения (18), (19) теоремь 3 (при прежнем определении (17) оценки $\theta^{*}$ ) сохранятся при

$$
\begin{aligned}
& \varphi_{(F)}=\min _{\lambda} \int_{-N}^{N-\Delta} f(x)\left(\frac{f(x)}{g(x)}\right)^{\lambda} d x+o(1)<1, \\
& \varphi_{(G)}=\min _{\lambda} \int_{-N}^{N-\Delta} g(x)\left(\frac{f(x)}{g(x)}\right)^{\lambda} d x+o(1)<1
\end{aligned}
$$

$\partial \bumpeq \Omega M \rightarrow \infty$ 
Аналогичные построения можно делать и в общем случае (с заменой интеграла, например в $\varphi_{(F)}$, на $\left.\int d F(x)\left(\frac{d F(x)}{d G(x)}\right)^{\lambda}\right)$, но в этом случае они будут выглядеть сложнее. Кроме того, возникают технические трудности, связанные с элементами выборки вне $[-N, N]$ при некотором $N$. Эти трудности можно устранить, если нам известно такое $N$, что сужения распределений $F$ и $G$ на $[-N, N]$ различны.

Таким образом, построения оченок $\theta^{*} c$ «собственной» ошибкой (сравнимой с 1) возможно, если предполагать лишь выполнение (2).

С другой стороны, если использовать только эмпирические функции распределения $F_{t}(x)$ и $F^{(n-t)}(x)$, построенные по выборкам $\mathrm{X}_{(t)}$ и $\mathrm{X}^{(n-t)}$ соответственно, и минимизировать по $t$ те или иные функционалы от этих функций, то получить точность для $\theta^{*}$ лучше, чем $O(\sqrt{n})$, невозможно. Действительно, названные эмпирические функции к моменту $t=\theta$, сравнимому с $n$ (см. условие (3)), набирают «шум» порядка $1 / \sqrt{n}$ и чтобы на этом фоне выделить изменение распределения, нужно порядка $\sqrt{n}$ или более наблюдений.

\section{2. Последовательное оценивание моментов разладки}

При рассмотрении последовательных процедур мы не будем фиксировать объем выборки $n$, считая его, если понадобится, неограниченным. Однако нам будет удобно сохранить растущий параметр $n$, который можно ассоциировать с большим возможным объемом наблюдений, и считать, что $\theta=\theta(n) \rightarrow \infty$ при $n \rightarrow \infty$. (Делать основным растушим параметром неизвестное $\theta$ не всегда удобно.)

2.1. Последовательное оценивание при выполнении условий $\left[\mathbf{h}_{\mathbf{1}}, \ldots, \mathbf{h}_{\mathbf{l}}\right]$. В условиях этого пункта в дополнение $\mathrm{\kappa}\left[\mathbf{h}_{\mathbf{1}}, \ldots, \mathbf{h}_{\mathbf{l}}\right]$ мы будем предполагать также, что нам известны значения

$$
a_{j}=\mathbf{E} h_{j}\left(\mathrm{x}^{(F)}\right), \quad j=1, \ldots, l,
$$

и постоянная $b>0$ такая, ито

$$
\max _{j}\left|\mathbf{E} h_{j}\left(\mathrm{x}^{(F)}\right)-\mathbf{E} h_{j}\left(\mathrm{x}^{(G)}\right)\right|>2 b .
$$

Другими словами, мы знаем, что максимальное ожидаемое изменение $\mathbf{E} h_{j}\left(\mathrm{x}^{(H)}\right)$ при переходе от $H=F$ к $H=G$ превосходит известное нам значение $2 b$.

Предположение о том, что значения $a_{j}$ нам известны, является естественным, так как «начальное» поведение наблюдаемой системы обычно известно. Если $a_{j}$ неизвестны, то при выполнении условия

$$
\theta>M
$$


(первого неравенства в условии (2)) значения $a_{j}$ можно оценить по первым $M$ наблюдениям величиной $M^{-1} \sum_{i=1}^{M} h_{j}\left(\mathrm{x}_{i}\right)$ (см. об этом далее замечание 3).

Условие (25) можно трактовать и иначе: нам неизвестна величина $b$ в (25), но мы можем указать «значимое» для эксперимента максимальное изменение $\mathbf{E} h_{j}\left(\mathrm{x}^{(H)}\right)$ (при замене $H=F$ на $H=G$ ), момент появления которого нам важно определить. Меньшие изменения для нас не существенны, их можно «не замечать» и считать, что в момент смены $F$ на $G$ (если таковая имела место) «разладки» в новом для нас понимании не произошло.

Отметим, что на самом деле такого рода «количественные» оговорки о существенности изменений должны присутствовать во всех задачах о разладке, так как небольшие изменения $F$ могут оставаться не замеченными при любой процедуре. Например, если в схеме серий $\rho(F, G)=O(1 / \sqrt{n})$, где $\rho$ - какая-нибудь метрика, то в течение $n$ наблюдений такое изменение с положительной вероятностью не будет выявлено даже при известных $F$ и $G$ и при использовании оптимальных процедур.

Итак, либо мы будем предполагать, что выполнено (25), либо мы сами назначаем величину $b$ и ищем такой момент разладки, при котором выполнено (25). При этом если (25) не выполнено, то момент смены $F$ на $G$ может оказаться не обнаруженным.

Иные подходы, не связанные с (25), будут рассмотрены в пп. 2.2, 2.3. При выполнении (25) рассмотрим $2 l$ случайных блужданий

$$
\mathrm{w}_{ \pm}^{(j)}(k)=\max \left[0, \mathrm{w}_{ \pm}^{(j)}(k-1) \pm\left(h_{j}\left(\mathrm{x}_{k}\right)-a_{j}\right)-b\right], \quad j=1, \ldots, l .
$$

Так как $\mathbf{E}\left[ \pm\left(h_{j}\left(\mathrm{x}^{(F)}\right)-a_{j}\right)-b\right]=-b<0$, то до момента разладки блуждания $\mathrm{w}_{ \pm}^{(j)}$ будут эргодическими. Если максимум в $(25)$ достигается на номере $j$ и

$$
\mathbf{E}\left[h_{j}\left(\mathrm{x}^{(G)}\right)-h_{j}\left(\mathrm{x}^{(F)}\right)\right] \geqslant 2 b
$$

то после момента разладки средний снос блуждания $\mathrm{w}_{+}^{(j)}$ будет удовлетворять неравенству

$$
\mathrm{E} h_{j}\left(\mathrm{x}^{(G)}\right)-a_{j}-b \geqslant b>0 .
$$

Если вместо (27) выполнено

$$
\mathbf{E}\left[h_{j}\left(\mathrm{x}^{(G)}\right)-h_{j}\left(\mathrm{x}^{(F)}\right)\right] \leqslant-2 b
$$

то средний снос блуждания $\mathrm{w}_{-}^{(j)}$ будет удовлетворять неравенству

$$
\mathbf{E}\left[-h_{j}\left(\mathrm{x}^{G)}\right)+a_{j}-b\right] \geqslant b>0 .
$$


Для простоты мы ограничимся рассмотрением случая, когда все случайные величинь $h_{j}\left(\mathrm{x}^{(F)}\right)$ удовлетворяют двустороннему условию Крамера и нам известно число $\mu>0$ такое, что

$$
\begin{gathered}
\mu<\min _{j} \lambda_{j}, \quad \lambda_{j}=\sup \left\{\lambda: \mathbf{E} e^{\lambda\left|h_{j}\left(\mathbf{x}^{(F)}\right)\right|}<\infty\right\} \\
\mathbf{E} \exp \left\{\mu\left[ \pm\left(h_{j}\left(\mathbf{x}^{(F)}\right)-a_{j}\right)-b\right]\right\}<1
\end{gathered}
$$

nри всех $j$. Тогда по аналогии с результатами [1] мы приходим к следующей естественной процедуре построения момента остановки, «ближайшего» к точке $\theta$ : наблюдения продолжаются до момента $\nu=\nu(N, d(k))$, когда впервыле произойдет хотя бы одно из событий

$$
\mathrm{w}_{ \pm}^{(j)}(k) \geqslant N+d(k)
$$

при некотором $N$ и $d(k)=\mu^{-1} \ln k, k=1,2, \ldots$. Другими словами, если

$$
\nu_{ \pm}^{(j)}=\min \left\{k: \mathrm{w}_{ \pm}^{(j)}(k) \geqslant N+d(k)\right\}
$$

TO

$$
\nu=\min \nu_{ \pm}^{(j)}
$$

Теорема 4. Пусть вылолнень условия $\left[\mathbf{h}_{\mathbf{1}}, \ldots, \mathbf{h}_{\mathbf{l}}\right],(24),(25),(30)$. Tогда:

1) выполнено неравенство

$$
\mathbf{P}_{\theta}\{\nu(N, d(\cdot))<\theta\} \leqslant 2 d l e^{-\mu_{1} N}
$$

$2 \partial e$

$$
\begin{gathered}
d=\sum_{k=1}^{\infty} k^{-\mu_{1} / \mu}, \quad \mu_{1}=\min _{j, \pm} \mu_{ \pm}^{(j)} \\
\mu_{ \pm}^{(j)}=\sup \left[\lambda: \exp \left\{\lambda\left[ \pm\left(h_{j}\left(\mathrm{x}^{(F)}\right)-a_{j}\right)-b\right]\right\} \leqslant 1\right], \quad \mu_{1}>\mu
\end{gathered}
$$

2) $п р и я \rightarrow \infty$

$$
\mathbf{E}_{\theta}(\nu-\theta \mid \nu>\theta)<\frac{N+\mu^{-1} \ln \theta}{b}(1+o(1)) .
$$

3 а м е ч а н и е 2 . Среднее значение «запаздывания» $\nu-\theta$ здесь имеет, как и в $[1]$, порядок $\ln \theta$, т.е. тот же порядок (с точностью до постоянного множителя), что и для асимптотически оптимальной процедуры при известных $F$ и $G$ (см. [10], [11]). 
Д ок а з а т л л с т в о т е о р е м ы совершенно аналогично доказательству теоремы 9 в [1]. Утверждение (31) вытекает из неравенств

$$
\begin{aligned}
\mathbf{P}_{\theta}\{\nu<\theta\} \leqslant & \mathbf{P}_{\theta}\left\{\bigcup_{j=1}^{l} \bigcup_{k=1}^{\theta-1}\left\{\mathrm{w}_{+}^{(j)}(k) \geqslant N+d(k)\right\}\right\} \\
& +\mathbf{P}_{\theta}\left\{\bigcup_{j=1}^{l} \bigcup_{k=1}^{\theta-1}\left\{\mathrm{w}_{-}^{(j)}(k) \geqslant N+d(k)\right\}\right\} \\
\leqslant & \sum_{j=1}^{l} \sum_{k=1}^{\infty} \mathbf{P}_{\infty}\left\{\mathrm{w}_{+}^{(j)}(k) \geqslant N+d(k)\right\} \\
& +\sum_{j=1}^{l} \sum_{k=1}^{\infty} \mathbf{P}_{\infty}\left\{\mathrm{w}_{-}^{(j)}(k) \geqslant N+d(k)\right\} .
\end{aligned}
$$

Но равномерно по $k$

$$
\mathbf{P}\left\{\mathrm{w}_{ \pm}^{(j)}(k) \geqslant v\right\} \leqslant e^{-\mu_{1} v}
$$

при всех $j$ (см., например, [12] и определение $\mu_{1}$ ). Поэтому

$$
\mathbf{P}_{\theta}\{\nu<\theta\} \leqslant 2 l \sum_{k=1}^{\infty} e^{-\mu_{1}(N+d(k))}=2 l d e^{-\mu_{1} N}
$$

Утверждение (32) доказывается столь же просто: после момента разладки в силу неравенств (28), (29) среднее время достижения границы $N+\mu^{-1} \ln (\theta+k)$ хотя бы одним из блужданий $\mathrm{w}_{ \pm}^{(j)}(\theta+k)$ не превзойдет в силу теоремы восстановления $b^{-1}\left(N+\mu^{-1} \ln \theta\right)(1+o(1))$. Теорема доказана.

3 а м е ч а н и е 3 . Если $a_{j}$ не известны и выполнено условие $(26)$, то для $a_{j}$ можно построить оценки

$$
a_{j}^{*}=M^{-1} \sum_{i=1}^{M} h_{j}\left(\mathrm{x}_{i}\right)
$$

Если для $h\left(\mathrm{x}_{j}^{(F)}\right)$ выполнено двустороннее условие Крамера, то

$$
\mathbf{P}\left\{\left|a_{j}^{*}-a_{j}\right|>M^{-1 / 4}\right\} \leqslant e^{-c \sqrt{M}}
$$

Такая оценка позволяет использовать описанную в теореме 4 процедуру при замене $a_{j}$ на $a_{j}^{*}$ и при добавлении в правой части неравенства (31) слагаемого $e^{-c \sqrt{M}}$. Если $\theta$ случайно, $P_{(M)}=\mathbf{P}\{\theta \leqslant M\} \rightarrow 0$ при $M \rightarrow \infty$, то при сохранении того же подхода в правой части (31) появится также слагаемое $P_{(M)}$. 


\section{2. Последовательное оценивание при известном распре-} делении $F$. В этом пункте мы будем предполагать, что $F$ известно. Случай неизвестного $F$ при выполнении (2) рассмотрен в п. 2.3.

Рассмотрим следующий общий подход. Разделим выборку Х на подвыборки $\mathrm{X}_{k m, m}=\left(\mathrm{x}_{k m+1}, \ldots, \mathrm{x}_{(k+1) m}\right)$ длиной $m$ и по каждой из них построим эмпирическую функцию распределения $F_{k, m}$. Выберем какоенибудь расстояние $\rho$ между распределениями, для которого известна асимптотика вероятности

$$
\mathbf{P}_{\theta}\left\{\sqrt{m} \rho\left(F, F_{m}\right)>z\right\} \quad \text { при } \quad z \rightarrow \infty, \quad z=o(\sqrt{m}),
$$

где $F_{m}=F_{1, m}, \theta>m$. Можно рассматривать, скажем, расстояния Колмогорова, $\sqrt{\chi^{2}}, \sqrt{\omega^{2}}$ и др. Например, для расстояния Колмогорова

$$
\rho\left(F, F_{m}\right)=\sup _{t}\left|F(t)-F_{m}(t)\right|
$$

при $z \rightarrow \infty, z=o(\sqrt{m})$ и непрерывной функции $F$ выполняется

$$
\mathbf{P}_{\theta}\left\{\sqrt{m} \rho\left(F, F_{m}\right)>z\right\} \sim 2 e^{-2 z^{2}} .
$$

Если $F$ произвольно, то при всех $m$ и $z$ имеет место неравенство

$$
\mathbf{P}_{\theta}\left\{\sqrt{m} \rho\left(F, F_{m}\right)>z\right\} \leqslant 2 e^{-2 z^{2}}
$$

(см. [13]). Для определенности мы остановимся на расстоянии Колмогорова. Отметим, что для метрики (33) и двух эмпирических функций распределения $F_{m_{1}}$ и $F_{m_{2}}$, соответствующих непрерывной $F$, справедливо также соотношение

$$
\mathbf{P}\left\{\sqrt{\frac{m_{1} m_{2}}{m_{1}+m_{2}}} \rho\left(F_{m_{1}}, F_{m_{2}}\right)>z\right\} \sim 2 e^{-2 z^{2}}
$$

при $z \rightarrow \infty, z^{4}=o\left(\left(m_{1}+m_{2}\right) \min \left(m_{1}, m_{2}\right)\right)$ (см. [14]). Это соотношение нам понадобится в п. 2.3 , где $F$ будет оцениваться по выборке.

Вернемся к случаю, когда $F$ известно. Для каждой из эмпирических функций распределения $F_{k, m}, k=1,2, \ldots$, найдем расстояния $\rho_{k}=$ $\rho\left(F, F_{k, m}\right)$. Введем в рассмотрение границу $b(k)=\sqrt{N+\ln k}$, где $N$ фиксировано или $N \rightarrow \infty, N=o(m)$, и будем проводить наблюдения до момента

$$
\eta=\min \left\{k: \sqrt{m} \rho_{k}>b(k)\right\},
$$

когда впервые произойдет событие $\left\{\sqrt{m} \rho_{k}>b(k)\right\}$. Если оно не произошло совсем в течение всех доступных наблюдений, то мы будем считать, что разладки не произошло. Если же событие (36) произошло, то мы будем утверждать, что

$$
\theta \in\left\{\begin{array}{lll}
{[1, m],} & \text { если } \quad \eta=1, \\
{[(\eta-2) m, \eta m],} & \text { если } \eta \geqslant 2 .
\end{array}\right.
$$


Вероятность ошибки (ложной тревоги) $P_{1}$ при этом будет удовлетворять при $m \rightarrow \infty$ соотношениям

$$
\begin{aligned}
P_{1}:=\mathbf{P}\{\eta<\infty \mid \theta=\infty\} & \leqslant \mathbf{P}\left\{\bigcup_{k=1}^{\infty}\left\{\sqrt{m} \rho_{k}>b(k)\right\} \mid \theta=\infty\right\} \\
& \leqslant \sum_{k=1}^{\infty} \mathbf{P}\left\{\sqrt{m} \rho_{k}>\sqrt{N+\ln k} \mid \theta=\infty\right\} \\
& \leqslant 2 \sum_{k=1}^{\infty} e^{-2(N+\ln k)} \leqslant 2 e^{-2 N} \sum_{k=1}^{\infty} e^{-2 \ln k}=c e^{-2 N}
\end{aligned}
$$

где $c=2 \sum_{k=1}^{\infty} k^{-2}<\infty$.

Значение $P_{1}$ есть аналог вероятности ошибки первого рода (разладки не было, а тревога прозвучала). Чтобы оценить аналог вероятности ошибки второго рода (разладка была, но осталась незамеченной), рассмотрим при условии

$$
A_{k}=\{\theta \in((k-1) m, k m]\}
$$

вероятность

$$
\begin{aligned}
P_{2}=\mathbf{P}\left\{\eta>k \mid A_{k}\right\} & \leqslant \mathbf{P}\left\{\sqrt{m} \rho_{k} \leqslant b(k) \mid A_{k}\right\} \\
& \leqslant \mathbf{P}\left\{\sqrt{m} \sup _{t}\left|G_{m}(t)-F(t)\right| \leqslant b\left(\frac{\theta}{m}+1\right)\right\}
\end{aligned}
$$

где $G_{m}(t)$ есть эмпирическая функция распределения, построенная по $m$ наблюдениям из распределения $G$. Последнее неравенство в (39) следует из того, что $A_{k}$ влечет за собой событие

$$
A_{k}^{\prime}=\left\{\text { все наблюдения из } \mathrm{X}_{k m, m} \text { имеют распределение } G\right\}, \quad k \geqslant 1 .(40)
$$

Если $N=o(m), \ln \theta=o(m)$ при $m \rightarrow \infty$, то

$$
b\left(\theta m^{-1}+1\right)=\sqrt{N+\ln (\theta+m)-\ln m}=o(\sqrt{m})
$$

и по теореме Санова (см. [15], [16]) в силу (39)

$$
\ln P_{2} \leqslant-m R(G, F)(1+o(1)),
$$

где $R(G, F)>0$ есть расстояние Кульбака-Лейблера между $G$ и $F$ :

$$
R(G, F)=\int\left[\ln \frac{d G(t)}{d F(t)}\right] d G(t) .
$$

Ясно также, что при всех $k$

$$
\begin{aligned}
P_{2} & =\mathbf{P}\{m \eta>m k \mid \theta \in((k-1) m, k m]\} \\
& \geqslant \mathbf{P}\{m \eta>\theta+m \mid \theta \in((k-1) m, k m]\} .
\end{aligned}
$$


Но оценка (42) от $k$ не зависит и, стало быть, для безусловной вероятности $\mathbf{P}_{\theta}\{m \eta>\theta+m\}$ будем иметь

$$
\ln \mathbf{P}_{\theta}\{m \eta>\theta+m\} \sim-m R(G, F)(1+o(1)) .
$$

Мы доказали следующее утверждение.

Теорема 5. Пусть $m \rightarrow \infty$ при $n \rightarrow \infty, \ln \theta=o(m)$, момент разладки оченивается с помощью решающего правила (37), где величина $\eta$ определена соотношением (36), $\rho_{k}=\rho\left(F, F_{k, m}\right)$. Тогда вероятности ошибок этого решающего правила обладают свойствами

$$
\begin{aligned}
\mathbf{P}\{\eta<\infty \mid \theta=\infty\} & \leqslant c e^{-2 N}, \\
\ln \mathbf{P}_{\theta}\{m \eta>\theta+m\} & \sim-m R(G, F),
\end{aligned}
$$

где с $=2 \sum_{k=1}^{\infty} k^{-2}, R$ определено в (43).

2.3. Последовательное оценивание при неизвестных $F$ и $G$ и при выполнении неравенства $\theta>M$. Если выполнено неравенство $\theta>M$ (условие (26)), то распределение $F$ можно оценивать по $M$ первым наблюдениям с помощью эмпирической функции распределения $F_{M}$. Остальные наблюдения, как и в предыдущем пункте, разобьем на подвыборки $\mathrm{X}_{M+k m, m}$ объема $m$. Эмпирические функции распределения, построенные по этим подвыборкам, вновь обозначим через $F_{k, m}$. Для упрощения изложения будем считать, что $M$ и $m$ имеют один порядок роста (они «соизмеримы»), т.е. $M / m \rightarrow r \in(0, \infty)$ при $n \rightarrow \infty$. Тогда, согласно (35), для непрерывных $F$

$$
\mathbf{P}\left\{q \sqrt{m} \rho\left(F_{M}, F_{k, m}\right)>z\right\} \sim 2 e^{-2 z^{2}}, \quad z \rightarrow \infty,
$$

где $q=\sqrt{r /(1+r)} \sim \sqrt{M /(M+m)}$. Если $F$ имеет скачки, то знак $\sim$ в (46) следует заменить на асимптотическое неравенство, так как при появлении разрывов у функции $F$ расстояние $\rho\left(F_{M}, F_{k, m}\right)$ уменьшается по распределению.

Последовательная процедура отыскания момента разладки в новых условиях совершенно аналогична изложенной в п. 2.2. Функции $b(k)$ и $\eta$ определяются как прежде (см. (36)) при замене $\rho_{k}$ на $\rho_{k}^{*}=q \rho\left(F_{M}, F_{k, m}\right)$. Решающее правило (37) остается в силе. В этом случае, как и прежде, в силу (46) при $N \rightarrow \infty$

$$
\begin{aligned}
P_{1}=\mathbf{P}\{\eta<\infty \mid \theta=\infty\} & \leqslant \sum_{k=1}^{\infty} \mathbf{P}\left\{\sqrt{m} \rho_{k}^{*}>\sqrt{N+\ln k} \mid \theta=\infty\right\} \\
& \leqslant 2 \sum_{k=1}^{\infty} e^{-2(N+\ln k)}(1+o(1))=c e^{-2 N}(1+o(1)) .
\end{aligned}
$$


Пусть $A_{k}=\{\theta \in(M+(k-1) m, M+k m]\}$ (ср. с (38)). Тогда для условной вероятности

$$
P_{2}=\mathbf{P}\left\{\eta>k \mid A_{k}\right\} \leqslant \mathbf{P}\left\{\sqrt{m} \rho_{k}^{*} \leqslant b(k) \mid A_{k}\right\}
$$

имеем

$$
\begin{aligned}
P_{2} \leqslant & \mathbf{P}\left\{\sqrt{M} \rho\left(F, F_{M}\right) \geqslant b(k) \sqrt{r} q^{-1} \mid A_{k}\right\} \\
& +\mathbf{P}\left\{\sqrt{m} \rho_{k}^{*} \leqslant b(k) \mid A_{k}, \sqrt{M} \rho\left(F, F_{M}\right)<b(k) \sqrt{r} q^{-1}\right\} .
\end{aligned}
$$

В силу неравенства треугольника $\rho\left(F_{k, m}, F\right) \leqslant \rho\left(F, F_{M}\right)+\rho\left(F_{M}, F_{k, m}\right)$. Поэтому

$$
\sqrt{m} \rho_{k}^{*} \geqslant q \sqrt{m} \rho_{k}^{\prime}-q \sqrt{m} \rho\left(F, F_{M}\right)
$$

где $\rho_{k}^{\prime}$ распределено как $\rho_{k}$ в п. 2.2 и не зависит от $\rho\left(F, F_{M}\right)$, так что второе слагаемое в правой части (48) при выполнении $A_{k}$ и при $\ln \theta=o(m)$ не превосходит

$$
\begin{aligned}
& \mathbf{P}\left\{q \sqrt{m} \sup \left|G_{m}(t)-F(t)\right| \leqslant 2 b(k)\right\} \\
& \quad \leqslant \mathbf{P}\left\{\sqrt{m} \sup \left|G_{m}(t)-F(t)\right| \leqslant \frac{2}{q} b\left(\frac{\theta-M+m}{m}\right)\right\} \\
& \quad \leqslant e^{-m R(G, F)(1+o(1))} .
\end{aligned}
$$

Следовательно, при $\ln \theta=o(m)$ в силу (47)-(49) (напомним, что $k m \geqslant$ $\theta-M$ на $\left.A_{k}\right)$

$$
\begin{aligned}
P_{2} & \leqslant 2 \exp \left\{-\frac{2 r}{q^{2}}\left(N+\ln \frac{\theta-M}{m}\right)\right\}(1+o(1))+e^{-m R(G, F)(1+o(1))} \\
& =2 e^{-2 r N / q^{2}}\left(\frac{\theta-M}{m}\right)^{-2 r / q^{2}}(1+o(1))+e^{-m R(G, F)(1+o(1))}
\end{aligned}
$$

Отсюда, аналогично предыдущему, мы получаем следующее утверждение.

Теорема 6. Пусть $m \rightarrow \infty, \ln \theta=o(m), M / m \rightarrow r \in(0, \infty)$ при $n \rightarrow \infty$; момент разладки оченивается с помощью решаюшего правила (37), где функиия $\eta$ определяется в (36) при замене $\rho_{k}$ на $\rho_{k}^{*}=q \rho\left(F_{M}, F_{k, m}\right), q=\sqrt{r /(1+r)}, N=o(m)$. Тогдa

$$
\begin{aligned}
\mathbf{P}\{\eta<\infty \mid \theta=\infty\} \leqslant & c e^{-2 N}(1+o(1)), \quad c=2 \sum_{k=1}^{\infty} k^{-2} \\
\mathbf{P}_{\theta}\{m \eta>\theta-M+m\} \leqslant & 2 e^{-2 r N / q^{2}}\left(\frac{\theta-M}{m}\right)^{-2 r / q^{2}} \\
& \times(1+o(1))+e^{-m R(G, F)(1+o(1))}
\end{aligned}
$$


3 а м е чан и е 4. Параметр $\theta$ в теоремах 5, 6 согласно правилу (37) определяется с точностью до $2 m$. Поэтому если $\theta$ и $n$ суть величины одного порядка, то в теоремах 5,6 следует брать «наименьшее» $m$, удовлетворяющее условию $m \gg \ln n$ (или $m \gg \ln \theta$ ). Это означает, что точность определения $\theta$ может иметь, например, порядок $m=(\ln \theta) \ln \ln \theta$. Это не сильно отличается от времени запаздывания в теореме 4 (см. замечание 2 ), имеющего порядок $\ln \theta$, как и в асимптотически оптимальной процедуре при известных $F$ и $G$. Если $m$ сравнимо с $\ln \theta$ и выбрано так, что

$$
m>(1+v) \frac{\ln \theta}{\rho^{2}(G, F)},
$$

где $v>0$ фиксировано, то в правых частях (45), (50) вместо $m R(G, F)$ будет стоять" $m Q(v)$, где $Q(v)>0$ при $v>0, Q(v) \rightarrow R(G, F)$ при $v \rightarrow \infty$. В этом случае порядок времени запаздывания в теоремах 5,6 будет $\ln \theta$ - такой же, как для асимптотически оптимальной процедуры в [10], [11] при известных $F$ и $G$. Однако $\theta$ и $\rho(F, G)$ нам не известны и указать в явном виде нужное нам $m$ в $(51)$ не удается.

2.4. О других подходах к последовательному оцениванию $\theta$. Отметим, что в литературе рассматриваются также алгоритмы последовательного отыскания $\theta$, допускающие частые ложные тревоги. Это соответствует тому, что в описанных выше процедурах растущая граница $N+d(k)$ в п. 2.1 и граница $b(k)=\sqrt{N+\ln k}$ в п. 2.2 заменяется постоянной границей $b(k)=N=$ const. Ясно, что тогда средняя задержка $\mathbf{E}_{\theta}(\nu-\theta \mid \nu>\theta)$, например, в (32) при больших фиксированных $N$ будет оцениваться значением $b^{-1} N(1+o(1))$ (и не зависеть от $\theta$ ), а среднее число ложных тревог будет расти вместе с $\theta$ линейно. Например, если выполнены условия теоремы 4, то среднее время между ложными тревогами будет оцениваться значением $\left(2 l e^{-\mu_{1} N}\right)^{-1}$ (ср. с $\left.(31)\right)$, если после каждой ложной тревоги вся процедура начинается заново. Если же начатая процедура продолжается, то оценки времени между ложными тревогами будут сложнее.

В [8] при выполнении условий $\left[\mathscr{X}_{1}, \ldots, \mathscr{X}_{l}\right]$ предложены именно такого рода последовательные процедуры, допускающие растущее вместе с $\theta$ число ложных тревог. Рассмотрены случаи, когда $F$ известно и когда оба распределения $F$ и $G$ неизвестны. Приведены схемы доказательства утверждений, в силу которых для некоторого большого параметра $N$ время задержки имеет порядок $N$, а среднее расстояние между ложными тревогами - порядок $e^{c N}, c=$ const.

Описанные процедуры с большим количеством ложных тревог требуют дополнительных исследований, связанных с оценкой временны́х и материальных затрат на проверку истинности тревог. Эти затраты 
растут пропорционально $\theta$ при $\theta \rightarrow \infty$. Если другие источники информации об истинности тревог, кроме наблюдений $\mathrm{x}_{i}$, отсутствуют, то возникает необходимость использовать наблюдения после каждой объявленной тревоги в таких количествах, что время задержки (до момента подтверждения истинности тревоги) составит величину порядка $\ln \theta$ (т.е. того же порядка, что и в теореме 4; предполагается, конечно, что при этом обеспечивается малость вероятности ошибочного подтверждения). Таким образом, в этом случае процедуры с частыми ложными тревогами во многом теряют свой смысл.

\section{СПИСОК ЛИТЕРАТУРЫ}

1. Borovkov A. A., Linke Yu. Yu. Change-point problem for large samples and incomplete information on distributions. - Math. Methods Statist., 2005, v. 14, № 4, p. 404-430.

2. Дарховский Б. С. Непараметрический метод для апостериорного обнаружения момента «разладки» последовательности независимых случайых величин. Теория вероятн. и ее примен., 1976, т. 21, в. 1, с. 180-184.

3. Carlstein E. Nonparametric change-point estimation. - Ann. Statist., 1988, v. 16, № 1, c. 188-197.

4. Hawkins D. L. A simple least squares method for estimating a change in mean. Comm. Statist. Simulation, 1986, v. 15, p. 655-679.

5. Dümbgen L. The asymptotic behavior of some nonparametric change-point estimators. - Ann. Statist., 1991, v. 19, № 3, p. 1471-1495.

6. Boukai B., Zhou H. Nonparametric estimation in a two change-point model. - J. Nonparametr. Statist., 1997, v. 8, № 3, p. 275-292.

7. Huang W.-T., Chang Y.-P. Nonparametric estimation in change-point models. J. Statist. Plann. Inference, 1993, v. 35, № 3, p. 335-347.

8. Baron M.I. Nonparametric adaptive change-point estimation and on-line detection. - Sequential Anal., 2000, v. 19, № 1-2, p. 1-23.

9. Brodsky B.E., Darkhovsky B.S. Nonparametric Methods in Change-point Problems. Dordrecht: Kluwer, 1993, 209 p.

10. Боровков $A . A$. Асимптотически оптимальные решения в задаче о разладке. Теория вероятн. и ее примен., 1998, т. 43, в. 4, с. 625-654.

11. Боровков A. А. Математическая статистика. Новосибирск: Наука, 1997, 771 с.

12. Боровков $A$. A. Вероятностные процессы в теории массового обслуживания. М.: Наука, 1972, 367 c.

13. Massart $P$. The tight constant in the Dvoretzky-Kiefer-Wolfowitz inequality. - Ann. Probab., 1990, v. 18, № 3, p. 1269-1283.

14. Боровков A. А. К задаче о двух выборках. - Изв. АН СССР, 1962, т. 26, № 4, c. $605-624$.

15. Санов И.Н. О вероятности больших уклонений случайных величин. - Матем. сб., 1957, т. 42, № 1, с. 11-44.

16. Боровков A.A. Граничные задачи для случайных блужданий и большие уклонения в функциональных пространствах. - Теория вероятн. и ее примен., 1967, т. 12 , в. 4 , с. $635-654$. 